\author{
WOJCIECH ZYGMUNT
}

\title{
On compactness and connectedness of the paratingent
}

\begin{abstract}
In this note we shall prove that for a continuous function $\varphi: \Delta \rightarrow$ $\mathbb{R}^{n}$, where $\Delta \subset \mathbb{R}$, the paratingent of $\varphi$ at $a \in \Delta$ is a non-empty and compact set in $\mathbb{R}^{n}$ if and only if $\varphi$ satisfies Lipschitz condition in a neighbourhood of $a$. Moreover, in this case the paratingent is a connected set.
\end{abstract}

1. Notations and definitions. Let $\mathbb{R}$ denote a real line, $\Delta \subset \mathbb{R}$ an interval and $\mathbb{R}^{n}$ the Euclidean $n$-dimensional space with usual norm

$$
\|x\|=\left(\sum_{i=1}^{n} x_{i}^{2}\right)^{1 / 2},
$$

where $x=\left(x_{1}, x_{2}, \ldots, x_{n}\right)$. The symbol

$$
\langle\langle A\rangle=\sup \{\|x\|: x \in A\}
$$

is defined for any $A \subset \mathbb{R}^{n}, A \neq \emptyset$. The differential quotient $\frac{\varphi(t)-\varphi(s)}{t-s}$, where $\varphi: \Delta \rightarrow \mathbb{R}^{n}$ is a continuous function, $t, s \in \Delta$ and $t<s$, is denoted by $\mathcal{D} q(t, s)$. Let $u^{\alpha}=(1-\alpha) t+\alpha \tau$ and $v^{\alpha}=(1-\alpha) s+\alpha \sigma$ for $t, s, \tau, \sigma \in \Delta$, $t<s, \tau<\sigma$ and $\alpha \in[0,1]$. Evidently, $u^{\alpha}<v^{\alpha}$.

The set of all points $x \in \mathbb{R}^{n}$ for which there exist two sequences $\left\{t_{k}\right\},\left\{s_{k}\right\}$ $\subset \Delta$ such that $t_{k}<s_{k}$, both sequences converge to $a$ and

$$
x=\lim _{k \rightarrow \infty} \mathcal{D} q\left(t_{k}, s_{k}\right),
$$

2010 Mathematics Subject Classification. 26A24, 54C50.

Key words and phrases. Paratingent, continuous function, Lipschitz condition. 
is called the paratingent of $\varphi$ at $a$ and is denoted by $(\mathcal{P} \varphi)(a)$.

We shall say that a function $\varphi: \Delta \rightarrow \mathbb{R}^{n}$ satisfies Lipschitz condition in a neighbourhood of a point $a \in \Delta$, if

$$
\exists_{L>0} \exists_{\delta>0} \forall_{t, s \in \Delta,|t-a|<\delta,|s-a|<\delta}\|\varphi(t)-\varphi(s)\| \leq L|t-s| .
$$

The distance of a point $x$ from set $A$ is denoted by

$$
\delta(x, A)=\inf \{\|x-y\|: y \in A\} .
$$

\section{Theorems.}

Theorem 2.1. The paratingent $(\mathcal{P} \varphi)(a)$ is a closed set in $\mathbb{R}^{n}$.

Proof. Let $x^{m} \in(\mathcal{P} \varphi)(a), m=1,2, \ldots$, and $\lim _{m \rightarrow \infty} x^{m}=x$. So we have

$$
x^{m}=\lim _{k \rightarrow \infty} \mathcal{D} q\left(t_{k}^{m}, s_{k}^{m}\right),
$$

where $t_{k}^{m}, s_{k}^{m} \in \Delta, t_{k}^{m}<s_{k}^{m}, \lim _{k \rightarrow \infty} t_{k}^{m}=\lim _{k \rightarrow \infty} s_{k}^{m}=a$ and $m=1,2, \ldots$

Then there exists $k_{m}$ for any $m$ such that $\left|t_{k_{m}}^{m}-a\right|<\frac{1}{m},\left|s_{k_{m}}^{m}-a\right|<\frac{1}{m}$, and $\left\|\mathcal{D} q\left(t_{k_{m}}^{m}, s_{k_{m}}^{m}\right)-x^{m}\right\|<\frac{1}{m}$. Hence

$$
x=\lim _{m \rightarrow \infty} \mathcal{D} q\left(t_{k_{m}}^{m}, s_{k_{m}}^{m}\right),
$$

where $\lim _{k \rightarrow \infty} t_{k_{m}}^{m}=\lim _{k \rightarrow \infty} s_{k_{m}}^{m}=a$. Thus $x \in(\mathcal{P} \varphi)(a)$, so $(\mathcal{P} \varphi)(a)$ is closed.

Theorem 2.2. The paratingent $(\mathcal{P} \varphi)(a)$ is a non-empty and compact set if and only if the function $\varphi$ satisfies Lipschitz condition in a neighbourhood of $a$.

Proof. $(\Leftarrow)$

Let $\varphi$ satisfy Lipschitz condition, hence there exist $L>0$ and $\delta>0$ such that $\|\mathcal{D} q(t, s)\| \leq L$ for any $t, s \in \Delta,|t-a|<\delta$ and $|s-a|<\delta$. Hence the paratingent $(\mathcal{P} \varphi)(a)$ is bounded. Thus, by Theorem $2.1,(\mathcal{P} \varphi)(a)$ is compact.

Let now $t_{k}, s_{k} \rightarrow a$ with $t_{k}<s_{k}$. The sequence $\left\{\mathcal{D} q\left(t_{k}, s_{k}\right)\right\}$ is bounded, so it contains a convergent subsequence, i.e. $\lim _{m \rightarrow \infty} \mathcal{D} q\left(t_{k_{m}}, s_{k_{m}}\right)=x \in$ $(\mathcal{P} \varphi)(a)$, hence $(\mathcal{P} \varphi)(a)$ is non-empty.

$(\Rightarrow)$

Let $(\mathcal{P} \varphi)(a)$ be non-empty and compact, and assume that $\varphi$ does not satisfy Lipschitz condition in any neighbourhood of $a$.

Firstly, let $x \in(\mathcal{P} \varphi)(a)$. There exists $M$ such that $\langle\langle(\mathcal{P} \varphi)(a)\rangle\rangle \leq M$. As $x$ belongs to $(\mathcal{P} \varphi)(a)$, we have $x=\lim _{k \rightarrow \infty} \mathcal{D} q\left(t_{k}, s_{k}\right)$ for some sequences $\left\{t_{k}\right\},\left\{s_{k}\right\} \subset \Delta, t_{k}<s_{k}$ and $t_{k}, s_{k} \rightarrow a$. Hence there exists $k_{0}$ such that $\left\|\mathcal{D} q\left(t_{k}, s_{k}\right)\right\|<2 M$ for $k \geq k_{0}$.

On the other hand, as $\varphi$ does not satisfy Lipschitz condition, there exist sequences $\left\{\tau_{k}\right\},\left\{\sigma_{k}\right\} \subset \Delta, \tau_{k}<\sigma_{k}$ and $\left|\tau_{k}-a\right|,\left|\sigma_{k}-a\right|<\frac{1}{k}$ such that $\left\|\mathcal{D} q\left(\tau_{k}, \sigma_{k}\right)\right\|>4 M$ for $k=1,2, \ldots$. 
Let now $\varrho_{k}(\alpha)=\left\|\mathcal{D} q\left(u_{k}^{\alpha}, v_{k}^{\alpha}\right)\right\|$, where $\alpha \in[0,1]$ and $u^{\alpha}, v^{\alpha}$ were defined in the first section. Function $\varrho_{k}:[0,1] \rightarrow \mathbb{R}$ is continuous and such that $\varrho_{k}(0)<2 M$ and $\varrho_{k}(1)>4 M$ for any $k=1,2, \ldots$. Thus there exists a sequence $\alpha_{k} \in[0,1]$ such that $\varrho_{k}\left(\alpha_{k}\right)=\left\|\mathcal{D} q\left(u_{k}^{\alpha_{k}}, v_{k}^{\alpha_{k}}\right)\right\|=3 M$.

Of course $u_{k}^{\alpha_{k}}, v_{k}^{\alpha_{k}} \rightarrow a$ as $k$ tends to infinity. The sequence of quotients $\mathcal{D} q\left(u_{k}^{\alpha_{k}}, v_{k}^{\alpha_{k}}\right)$ is bounded, hence it contains a subsequence $\mathcal{D} q\left(u_{k_{m}}^{\alpha_{k_{m}}}, v_{k_{m}}^{\alpha_{k_{m}}}\right)$ convergent to a point $y \in(\mathcal{P} \varphi)(a)$. But we have $\|y\|=3 M$, which contradicts the assumption $\|y\| \leq M$ as $(\mathcal{P} \varphi)(a)$ is bounded by the constant $M$. Therefore $\varphi$ must satisfy Lipschitz condition in some neighbourhood of $a$.

Theorem 2.3. If $\varphi: \Delta \rightarrow \mathbb{R}^{n}$ satisfies Lipschitz condition in a neighbourhood of $a \in \Delta$, then the paratingent $(\mathcal{P} \varphi)(a)$ is a continuum, i.e. it is a non-empty compact and connected set.

Proof. By Theorem 2.2 it is enough to show that $(\mathcal{P} \varphi)(a)$ is connected.

Assume "a contrario" that $(\mathcal{P} \varphi)(a)$ is not connected, i.e. $(\mathcal{P} \varphi)(a)=E_{0} \cup$ $E_{1}$, where sets $\emptyset \neq E_{i}, i=0,1$ are compact and $E_{0} \cap E_{1}=\emptyset$. Then $d=\inf \left\{\|x-y\|: x \in E_{0}, y \in E_{1}\right\}>0$.

Let $g: \mathbb{R}^{n} \rightarrow \mathbb{R}$ be a function given by the formula $g(x)=\delta\left(x, E_{0}\right)-$ $\delta\left(x, E_{1}\right)$. Function $g$ is continuous. Moreover, if $x \in E_{0}$, then $g(x) \leq-d$, and if $x \in E_{1}$, then $g(x) \geq d$. Hence $g(x) \neq 0$ for all $x \in(\mathcal{P} \varphi)(a)$.

Let us now fix $x^{0} \in E_{0}$ and $x^{1} \in E_{1}$. So we have $x^{0}=\lim _{k \rightarrow \infty} \mathcal{D} q\left(t_{k}, s_{k}\right)$ and $x^{1}=\lim _{k \rightarrow \infty} \mathcal{D} q\left(\tau_{k}, \sigma_{k}\right)$ for some sequences $\left\{t_{k}\right\},\left\{s_{k}\right\},\left\{\tau_{k}\right\},\left\{\sigma_{k}\right\} \subset \Delta$, $t_{k}<s_{k}, \tau_{k}<\sigma_{k}$ and

$$
\lim _{k \rightarrow \infty} t_{k}=\lim _{k \rightarrow \infty} s_{k}=\lim _{k \rightarrow \infty} \tau_{k}=\lim _{k \rightarrow \infty} \sigma_{k}=a
$$

There exists $k_{0}$ such that $g\left(\mathcal{D} q\left(t_{k}, s_{k}\right)\right)<-\frac{d}{2}$ and $g\left(\mathcal{D} q\left(\tau_{k}, \sigma_{k}\right)\right)>\frac{d}{2}$ for $k \geq k_{0}$.

Let us now consider a family of functions $h_{k}:[0,1] \rightarrow \mathbb{R}$, for $k \geq k_{0}$, given by the formula $h_{k}(\alpha)=g\left(\mathcal{D} q\left(u_{k}^{\alpha}, v_{k}^{\alpha}\right)\right)$. We have $h_{k}(0)=g\left(\mathcal{D} q\left(t_{k}, s_{k}\right)\right)<$ $-\frac{d}{2}<0$ and $h_{k}(1)=g\left(\mathcal{D} q\left(\tau_{k}, \sigma_{k}\right)\right)>\frac{d}{2}>0$. There exists a sequence $\alpha_{k} \in[0,1]$ such that $h_{k}\left(\alpha_{k}\right)=0$ for $k \geq k_{0}$. The sequence $\mathcal{D} q\left(u_{k}^{\alpha_{k}}, v_{k}^{\alpha_{k}}\right)$ is bounded, so it contains a subsequence $\mathcal{D} q\left(u_{k_{m}}^{\alpha_{k_{m}}}, v_{k_{m}}^{\alpha_{k_{m}}}\right)$ convergent to point $y \in(\mathcal{P} \varphi)(a)=E_{0} \cup E_{1}$. Hence $g(y) \neq 0$, which contradicts the fact that

$$
\begin{aligned}
g(y) & =g\left(\lim _{m \rightarrow \infty} \mathcal{D} q\left(u_{k_{m}}^{\alpha_{k_{m}}}, v_{k_{m}}^{\alpha_{k_{m}}}\right)\right)=\lim _{m \rightarrow \infty} g\left(\mathcal{D} q\left(u_{k_{m}}^{\alpha_{k_{m}}}, v_{k_{m}}^{\alpha_{k_{m}}}\right)\right) \\
& =\lim _{m \rightarrow \infty} h_{k_{m}}\left(\alpha_{k_{m}}\right)=0 .
\end{aligned}
$$

Therefore, the set $(\mathcal{P} \varphi)(a)$ is connected, which completes the proof. 
3. Remarks. The definition of paratingent used in this note is an analytic modification by A. Bielecki [2] of the original definition given by G. Bouligand [3]. The Bouligand definition has a geometrical character and it applies to every general set $E \subset \mathbb{R}^{n}$. Let us recall this definition (cf. [4, Def. VII.1.1]):

Definition. In the Euclidean space $\mathbb{R}^{n}$ the direction of a half-line (or in other words a ray $x y \rightarrow$ ) with origin at a point $x$ and passing through a point $y$ is identified in the well-known way with a point of the unit sphere in $\mathbb{R}^{n}$. This identification gives us the topological structure in the set of all directions (i.e. rays).

Paratingent of the set $E \subset \mathbb{R}^{n}$ at point $x \in E$ is the set $\left(\mathcal{P}_{E}\right)(x)$ of all limits of the directions of sequences of half-lines $y_{k} z_{k}$, where $y_{k}, z_{k} \in E$ and $y_{k}, z_{k} \rightarrow x$.

If a point $x$ is an accumulation point of the set $E$, then the paratingent $\left(\mathcal{P}_{E}\right)(x)$ is always compact and non-empty set (cf. [4, Proposition VII.1.2]). So let $\varphi: \Delta \rightarrow \mathbb{R}^{n}$ be a given continuous function. Then the paratingent in the Bouligand sense of the function $\varphi$ at point $a \in \Delta$ is the set $\left(\mathcal{P}_{\mathrm{Gr} \varphi}\right)((a, \varphi(a)))$, where $\operatorname{Gr} \varphi=\{(t, \varphi(t)): t \in \Delta\} \subset \mathbb{R}^{1+n}$ is the graph of the function $\varphi$. Of course the set $\left(\mathcal{P}_{\mathrm{Gr} \varphi}\right)((a, \varphi(a)))$ is always non-empty and compact in $\mathbb{R}^{n+1}$.

Instead, the paratingent presented in this note (i.e. in Bielecki sense) of a function $\varphi$ at a point $a$, i.e. the set $(\mathcal{P} \varphi)(a) \subset \mathbb{R}^{n}$, can be empty, bounded or unbounded.

Examples: Let $\varphi: \mathbb{R} \rightarrow \mathbb{R}$.

(1) $\varphi(t)=t^{1 / 3}$, then $(\mathcal{P} \varphi)(0)=\emptyset$, but

$$
\left(\mathcal{P}_{\mathrm{Gr} \varphi}\right)(0, \varphi(0))=\{(0,-1),(0,1)\} \subset \mathbb{R}^{2} ;
$$

(2) $\varphi(t)=|t|$, then $(\mathcal{P} \varphi)(0)=[-1,1] \subset \mathbb{R}$, but

$$
\left(\mathcal{P}_{\mathrm{Gr} \varphi}\right)(0, \varphi(0))=\left\{(\cos t, \sin t): t \in\left[-\frac{\pi}{4}, \frac{\pi}{4}\right] \cup\left[\frac{3}{4} \pi, \frac{5}{4} \pi\right]\right\} \subset \mathbb{R}^{2} ;
$$

(3) $\varphi(t)=\sqrt{|t|}$, then $(\mathcal{P} \varphi)(0)=\mathbb{R}$, but

$$
\left(\mathcal{P}_{\mathrm{Gr} \varphi}\right)(0, \varphi(0))=\{(\cos t, \sin t): t \in[0,2 \pi]\} \subset \mathbb{R}^{2} .
$$

In the literature (cf. $[1,5,6])$ the paratingent was considered only as a set-valued function acting from $\mathbb{R}$ into a family of non-empty subsets of $\mathbb{R}^{n}$. Instead in this note we characterize the set $(\mathcal{P} \varphi)(a)$ by the properties of $\varphi$.

Acknowledgement. The author is grateful to the anonymous referee for his/her helpful suggestions. 


\section{REFERENCES}

[1] Aubin, J. P., Frankowska, H., Set-Valued Analysis, Birkhauser, Boston, Massachusetts, 1990.

[2] Bielecki, A., Sur certaines conditions nécessaires et suffisantes pour l'unicité des solutions des systèmes d'équations différentielles ordinaires et des équations au paratingent, Ann. Univ. Mariae Curie-Skłodowska Sect. A 2 (1948), 49-106.

[3] Bouligand, G., Introduction à la géometrie infinitésimale directe, Vuibert, Paris, 1932.

[4] Choquet, G., Outils topologiques et métriques de l'analyse mathématique, Centre de Documentation Univ., Course rédigé par C. Mayer, Paris, 1969.

[5] Fedor, M., Szyszkowska, J., Darboux properties of the paratingent, Ann. Univ. Mariae Curie-Skłodowska Sect. A 62 (2008), 67-74.

[6] Mirica, S., The contingent and the paratingent as generalized derivatives for vectorvalued and set-valued mappings, Nonlinear Anal. 6 (1982), 1335-1368.

Wojciech Zygmunt

Faculty of Mathematics, Informatics and Landscape Architecture, KUL

al. Racławickie 14

20-950 Lublin

Poland

e-mail: wzygmunt@kul.lublin.pl

Received September 8, 2016 